\title{
Importancia del apoyo social en la permanencia de la abstinencia del consumo de drogas
}

\author{
María Luisa G armendia ${ }^{1}$, María Elena Alvarado ${ }^{1}$, \\ Mariano Montenegro², Paulina Pino¹. \\ Social support as a protective \\ factor of recurrence after \\ drug addiction treatment
}

\begin{abstract}
Background: Lack of social support can be one of the factors that influences recurrences of drug consumption after treatment of addictions. Aim: To assess the role of social support in maintaining drug abstinence after treatment. Ma terial and methods: We studied 306 subjects that were treated in drug addiction centers, financed by the National Council for Drug Control (CONACE). At discharge, social and demographic data were recorded and the Medical Outcomes Study (MOS) questionnaire was given to evaluate social support. Subjects that achieved abstinence at the moment of discharge were contacted six months later and interrogated about eventual drug consumption thereafter. Results: One hundred fifty three (76\% male, aged $32 \pm 10$ years) of 197 abstinent subjects at discharge, were located six months later. Of these, $108(71 \%)$ were not consuming drugs. On univariate analysis, social support had a protective effect against recurrence of drug consumption (OR - 0.98; CI 95\% $=0.96$ 0.99). This effect remained significant after adjusting for age, sex, occupational situation, mental health self-assessment, family history of alcohol and drug consumption, type of drug treatment and type of discharge as confounding variables (OR $=0.97$; CI 95\% $=0.94-0.99$ ). Conclusions: These data provide evidence that social support protects against recurrence into drug consumption at least up to six months. Long-term effects should be evaluated (Rev Méd Chile 2008; 136; 169-78).
\end{abstract}

(Key w ords: Mental health; Social support; Substance-related disorders)

Recibido el 11 de mayo, 2007. Aceptado el 31 de agosto, 2007.

Proyecto original diseñado y financiado por Consejo Nacional de Control de Estupefacientes, CONACE.

${ }^{1}$ Escuela de Salud Pública, Facultad de Medicina, Universidad de Chile, Santiago de Chile. ${ }^{2}$ Consejo Nacional de Control de Estupefacientes (CONACE), Ministerio del Interior, Gobierno de Chile.

Correspondencia a: Dra. María Luisa Garmendia. Escuela de Salud Pública, Facultad de Medicina, Universidad de Chile. Independencia 939, Santiago, Chile. Teléfono: 5629786142. E mail: mgarmend@med.uchile.cl 
$\mathrm{E}^{1}$ abuso y la dependencia de consumo de drogas constituyen un problema de salud pública en Chile, así como en la mayoría de los países de América Latina y del mundo occidental, tanto por su magnitud como por sus consecuencias a nivel social, político y económico. Las estrategias para enfrentar este problema han abordado, por una parte, la prevención del consumo y por otra, el tratamiento de las personas ya afectadas. Los tratamientos no se agotan en el logro de la abstinencia o la disminución del consumo, sino también se dirigen a la esfera de la salud física y mental y de una mejor inserción social en los ámbitos laboral, familiar y educacional ${ }^{1}$. El cuerpo de evidencia publicada está más centrado en la consecución de estos logros al finalizar el tratamiento, pero existe poca evidencia respecto a los factores que, una vez lograda la abstinencia del consumo, puedan favorecer o dificultar la permanencia de la misma ${ }^{2-4}$.

Uno de los factores que podría estar relacionado con la no reincidencia del consumo es el apoyo social. Este es definido como un proceso interactivo, gracias al cual, el individuo obtiene ayuda emocional, instrumental y económica de la red social en la que se encuentra inserto ${ }^{5-7}$. Numerosos estudios demuestran el impacto que ejerce el apoyo social percibido sobre los procesos relacionados con la salud y la enfermedad, así como de su efecto beneficioso sobre la evolución de enfermedades tan dispares como la depresión, artrosis o diabetes ${ }^{7-9}$. La manifestación de apoyo social, es considerada como un factor importante para prevenir o mitigar el efecto negativo de los acontecimientos vitales estresantes, tanto de forma directa como mediante un mecanismo tampón 0 amortiguador, $8,10-12$. Se han descrito cuatro tipos de apoyo social: a) emocional, que comprende la empatía, el cuidado, el amor y la confianza; b) instrumental, caracterizado por conductas específicas que directamente ayudan a quien lo necesita; c) informativo, que provee conocimientos a las personas afectadas para que éstas puedan enfrentar situaciones problemáticas y d) evaluativo, que implica sólo transmisión de información ${ }^{13}$.

El instrumento MOS, desarrollado por Sherbourne y cols en pacientes participantes del Medical Outcomes Study, es un cuestionario largamente utilizado en la evaluación del apoyo social en pacientes con enfermedades crónicas y ha mostra- do buenas propiedades psicométricas evaluando distintas dimensiones del apoyo social ${ }^{14}$. Es breve, de fácil comprensión y autoadministrado ${ }^{14,15}$.

El presente estudio pretende evaluar la importancia del apoyo social en la recaída a los seis meses del consumo de drogas en personas que lograron la abstinencia.

\section{MATERIAL Y MÉTODO}

Se estudió a una cohorte cuya característica común es el logro de la abstinencia de consumo previo a su egreso del tratamiento. En este caso, se trata de usuarios egresados de programas de tratamiento ambulatorios y residenciales de centros públicos y privados de las regiones Quinta, Sexta y Metropolitana de Chile, financiados por el Consejo Nacional para el Control de Estupefacientes (CONACE), organismo dependiente del Ministerio del Interior del Gobierno de Chile. La recolección de información se realizó entre julio de 2005 y abril de $2006^{16}$.

Los 52 centros en estudio constituyen una muestra de centros de tratamiento en convenio con CONACE-FONASA, seleccionada por un muestreo estratificado en base a la modalidad de tratamiento entregado (ambulatorio básico, ambulatorio intensivo y residencial) y número de pacientes atendidos por centro. De acuerdo a ese esquema de muestreo, se incluyó en forma secuencial a 306 personas egresadas, ya sea por alta terapéutica o por abandono del programa. De éstas, al momento del egreso, 197 (64\%) habían logrado la abstinencia. A los seis meses, se pudo recontactar a 153 personas que en definitiva constituyeron la muestra de este estudio.

Las principales causas de las 44 pérdidas de seguimiento fueron el abandono del hogar $(\mathrm{n}=17)$, la no posibilidad de contacto con los encuestados por cambios de domicilio o datos de contacto erróneos $(n=13)$ y el rechazo a seguir participando $(n=11)$. A fin de evaluar el sesgo potencial de estas pérdidas, se efectuó un análisis del perfil de las personas no ubicadas, el cual no detectó diferencias en las principales variables de estudio, incluido el puntaje en apoyo social (datos no mostrados).

$\mathrm{Al}$ momento del egreso del programa de tratamiento, los participantes - previa firma de consentimiento informado- respondieron un cuestionario 
estructurado aplicado por un encuestador entrenado. Esta encuesta contenía preguntas referentes a los siguientes ámbitos: a) perfil sociodemográfico tales como edad, sexo, estado civil, nivel educacional, situación ocupacional antes del tratamiento y al egreso; b) patrón de consumo de drogas antes del tratamiento; esto es, edad de inicio, tipo de droga, frecuencia y droga que motivó el ingreso al tratamiento; c) antecedentes de consumo problemático de alcohol y drogas en la familia, y d) la participación en actividades ilegales, antes del tratamiento y al egreso. Conjuntamente se evaluó la percepción del estado de salud mental mediante el cuestionario de salud general de Goldberg de 12 ítems (GHQ-12) ${ }^{17}$. Este es un cuestionario autoadministrado, desarrollado con el fin de cribar trastornos mentales actuales ${ }^{18,19}$ y que ha sido previamente validado en Chile por Araya, encontrando una sensibilidad y especificidad de $76 \%$ y $73 \%$, respectivamente ${ }^{20}$. Puntúa en un rango de 0 a 12 , siendo que a mayor puntaje, peor es el nivel de salud mental autopercibida ${ }^{17}$.

Esta variable se consideró como variable continua y también se categorizó en tres grupos: ausencia de psicopatología ( 0 a 4), patología subumbral ( 5 a 6) y presencia de psicopatología (7 a 12) ${ }^{19}$.

Finalmente, a partir de la ficha clínica, se consignó el tipo de tratamiento recibido (ambulatorio o residencial) y el diagnóstico clínico del paciente, agrupado en: abuso, dependencia, abuso más patología psiquiátrica y dependencia más patología psiquiátrica. La condición de egreso del programa de tratamiento se clasificó como alta terapéutica, abandono, derivación a otro centro y alta disciplinaria o expulsión.

Para evaluar el grado de apoyo social -nuestra variable de exposición-, se utilizó el cuestionario autoadministrado MOS, en su versión adaptada y validada en España, previamente utilizada en Chile $^{8,21}$, la cual permite investigar el apoyo global y sus cuatro dimensiones: 1) afectiva (demostración de amor, cariño y empatía); 2) de interacción social positiva (posibilidad de contar con personas para comunicarse); 3) instrumental (posibilidad de ayuda doméstica), y 4) emocional/ informacional (posibilidad de asesoramiento, consejo, información $)^{22}$. Está constituido por 20 ítems; el primero, dimensiona el número de amigos íntimos y familiares cercanos que tiene el entrevistado; los demás se estructuran de acuendo a escala de Likert que puntúa de 1 (nunca) a 5 (siempre) $)^{8,22-24}$. El índice global puntúa en un rango de 19 a 95, siendo mayor el apoyo social percibido cuanto mayor es este puntaje.

Al término de seis meses, los participantes fueron nuevamente contactados y recibieron una segunda visita del encuestador, ocasión en la que fueron consultados respecto a reincidencias en el consumo de drogas durante el período, la cual constituye la variable respuesta del estudio.

Se realizó un análisis exploratorio de todas las variables, incluyendo distribuciones de frecuencias y medidas clásicas de resumen. Se elaboró un modelo conceptual basado en grafos acíclicos dirigidos ${ }^{25,26}$, que permitió identificar como variables confusoras relevantes a la edad, sexo, situación ocupacional, autopercepción de salud mental (Goldberg), antecedente de consumo de drogas o alcohol en la familia, tipo de tratamiento recibido y tipo de egreso. Es decir, estas variables podrían explicar parcial o totalmente el eventual efecto del apoyo social sobre la recaída en el consumo de drogas, por lo que se requerirá un modelo ajustado para una mejor estimación del efecto.

La asociación de cada variable con el consumo de drogas a los seis meses del egreso (en agrupación dicotómica), se analizó a través de modelos de regresión logística no condicional univariados obteniéndose los respectivos Odds Ratios (OR) e intervalos de confianza de 95\% (IC 95\%). La importancia del apoyo social como factor predictivo de la recaída del consumo, se evaluó a partir de dos modelos multivariados de regresión logística no condicional: 1) un modelo que consideró la dimensión cuantitativa, es decir, el número de amigos íntimos y familiares cercanos y 2) un modelo en el cual la variable predictora fue el puntaje total de la escala MOS como variable continua. Ambos modelos incluyeron las variables identificadas como confusoras en la relación apoyo social-consumo, a fin de obtener estimadores ajustados. Para el análisis estadístico se utilizó el programa Stata 8.0 (Stata Statistical Software, Texas, USA).

\section{RESULTADOS}

La Tabla 1 presenta las características de la población en estudio. Mayoritariamente fueron hombres (76\%), con una edad promedio de 32 años e 
Tabla 1. Caracterización sociodemográfica e historia de consumo de drogas de la muestra de usuarios que lograron abstinencia al egreso de tratamiento

\begin{tabular}{|c|c|c|c|c|}
\hline & $\mathrm{N}$ & $\%$ & Promedio & $\begin{array}{c}\text { Desviación } \\
\text { estándar }\end{array}$ \\
\hline Edad (años) & 153 & & 31,8 & 9,9 \\
\hline Hombres & 116 & 75,8 & & \\
\hline Escolaridad (años) & 153 & & 10,6 & 2,9 \\
\hline Ocupación antes del tratamiento & 152 & & & \\
\hline Estudiando o con trabajo estable & 87 & 57,2 & & \\
\hline Desempleado o con trabajo ocasional & 61 & 40,1 & & \\
\hline Otros & 4 & 2,6 & & \\
\hline \multicolumn{5}{|l|}{ Edad inicio consumo drogas (años) } \\
\hline Alcohol & 121 & & 16,3 & 5,3 \\
\hline Marihuana & 84 & & 15,6 & 4,7 \\
\hline Cocaína & 90 & & 24,3 & 8,4 \\
\hline Pasta base & 69 & & 23,2 & 8,0 \\
\hline \multicolumn{5}{|l|}{ Droga motivo ingreso tratamiento } \\
\hline Alcohol & 36 & 23,5 & & \\
\hline Marihuana & 7 & 4,6 & & \\
\hline Pasta base & 61 & 39,9 & & \\
\hline Cocaína & 42 & 27,5 & & \\
\hline Consumo problemático de alcohol en la familia & 119 & 77,8 & & \\
\hline Consumo drogas ilegales en la familia & 92 & 60,9 & & \\
\hline Participación actividades ilegales vida & 148 & 43,2 & & \\
\hline \multicolumn{5}{|l|}{ Diagnóstico clínico } \\
\hline Abuso & 55 & 35,9 & & \\
\hline Dependencia & 51 & 33,3 & & \\
\hline Abuso con patología psiquiátrica & 7 & 4,6 & & \\
\hline Dependencia con patología psiquiátrica & 15 & 10,0 & & \\
\hline No consignado & 25 & 16,3 & & \\
\hline
\end{tabular}

iniciaron el consumo de drogas ilegales entre los 15 y 24 años, según el tipo de droga. La droga que más motivó el ingreso al tratamiento fue el consumo de pasta base de cocaína (40\%). Se destaca la alta prevalencia de consumo problemático de alcohol y drogas en la familia que alcanzó a 78\% y $61 \%$, respectivamente. El diagnóstico de abuso o dependencia fue proporcionalmente similar (alrededor de 34\%). El diagnóstico de comorbilidad psiquiátrica se consignó sólo en $15 \%$ y no se encontró registro de alguna categoría diagnóstica en la ficha clínica en $16 \%$ de los pacientes.

Otras características al egreso, además de la abstinencia de consumo se muestran en la Tabla 2. El tiempo promedio en tratamiento fue de 5,6 meses y poco más de un tercio abandonó volunta- riamente el tratamiento. El puntaje promedio en el GHQ-12 fue de 1,5, indicativo de buena salud mental. La participación en actividades ilegales en el último mes fue de $2 \%$. En cuanto al apoyo social percibido, el promedio de amigos íntimos y familiares cercanos fue de 7,2 y el índice global promedio en la escala MOS en los ítems de escala Likert fue de 78,9, con un rango que osciló entre 30 y 95.

Al sexto mes de seguimiento, 108 sujetos (70,6\%) aún se mantenían abstemios. Un primer análisis reveló que el egreso por abandono fue un factor de riesgo importante en la recaída del consumo (OR =2,39; IC 95\% =1,12-5,14) junto con el ingreso a tratamiento por consumo de pasta base de cocaína, que mostró cinco veces más riesgo respecto a otras drogas (OR $=5,44$; IC 95\% 

Tabla 2. Caracterización de logros alcanzados al egreso del tratamiento en usuarios
que lograron abstinencia del consumo al egreso de tratamiento

\begin{tabular}{|c|c|c|c|c|}
\hline & $\mathrm{N}$ & $\%$ & Promedio & $\begin{array}{c}\text { Desviación } \\
\text { estándar }\end{array}$ \\
\hline \multicolumn{5}{|l|}{ Tipo de tratamiento recibido } \\
\hline Ambulatorio básico & 57 & 68,7 & & \\
\hline Ambulatorio intensivo & 17 & 20,5 & & \\
\hline Residencial & 9 & 10,8 & & \\
\hline Tiempo en tratamiento (meses) & 146 & & 5,6 & 2,8 \\
\hline \multicolumn{5}{|l|}{ Tipo de egreso } \\
\hline Alta terapéutica & 87 & 56,9 & & \\
\hline Abandono & 52 & 34,0 & & \\
\hline Derivación & 13 & 8,5 & & \\
\hline Expulsión & 1 & 0,7 & & \\
\hline Participación en actividades ilegales & 151 & 2,0 & & \\
\hline Estudiar o trabajar en forma estable & 152 & 56,6 & & \\
\hline \multicolumn{5}{|l|}{ Puntaje Cuestionario salud general Goldberg } \\
\hline Continua & 152 & & 1,5 & 2,0 \\
\hline Ausencia psicopatología (0-4) & 136 & 89,5 & & \\
\hline Patología subumbral (5-6) & 10 & 6,6 & & \\
\hline Presencia psicopatología (7-12) & 6 & 4,0 & & \\
\hline Número de amigos íntimos y familiares cercanos (MOS) & 146 & & 7,2 & 5,2 \\
\hline Índice Global Apoyo Social (MOS) & 152 & & 78,9 & 15,6 \\
\hline
\end{tabular}

$=1,12-26,37)$. Una baja percepción del nivel de salud mental también se mostró asociada al riesgo del consumo (OR =1,20; IC 95\% =1,01-1,42). Entre los factores protectores del consumo, se encontró la mayor duración del tratamiento $(\mathrm{OR}=0,86$; $\mathrm{IC}$ $95 \%=0,75-0,98)$ y tener una ocupación estable, sea ésta trabajo o estudio (OR $=0,49$; IC 95\% $=0,25-1,00$ ) (Tabla 3).

La Tabla 4 muestra la importancia del apoyo social como factor predictor del consumo de drogas a los seis meses controlando el efecto de las demás variables. El índice global de apoyo social, mostró un efecto protector en el modelo univariado ( $\mathrm{OR}=0,98$; IC $95 \%=0,96-0,99$ ). Este efecto persiste tras ajustar por las demás variables potencialmente confusoras en esta relación (OR $=0,97 ;$ IC $95 \%=0,95-0,99)$. Es decir, cada punto de incremento en la escala de autopercepción de apoyo social, implica una disminución en $3 \%$ del OR -como estimador de riesgo- de recaída en el consumo. La dimensión estrictamente cuantitativa del cuestionario MOS - esto es, la cantidad percibida de amigos íntimos y familiares cercanos- no se mostró significativamente asociada con la recaída en el consumo.

\section{Discusión}

Este estudio confirmó la hipótesis de que el apoyo social es un factor protector de la recaída del consumo de drogas, en pacientes que habían alcanzado la abstinencia del consumo, tras seis meses de tratamiento. Se encontró que por cada punto de incremento en la escala de autopercepción de apoyo social, el riesgo de recaída disminuye en tres puntos porcentuales. Estos resultados corroboran lo encontrado por otros autores que sustentan la hipótesis de que el apoyo familiar o social diferencian a consumidores de no consumidores $^{27-29}$. Rhoads encontró, en individuos en tratamiento por adicción a heroína, que en aquellos que disponían de más soporte social disminuyó significativamente el consumo de heroína y otras drogas duras, así como los niveles de depresión y ansiedad ${ }^{30}$. Se han descrito dos 
Tabla 3. Variables asociadas al consumo de drogas a los seis meses en usuarios que lograron la abstinencia del consumo al egreso del tratamiento

\begin{tabular}{|c|c|c|c|}
\hline & Odds Ratio crudo & $\begin{array}{c}\text { Intervalo de confianza } \\
\text { de } 95 \%\end{array}$ & valor $\mathrm{p}$ \\
\hline Sexo Masculino & 0,98 & $0,44-2,20$ & 0,961 \\
\hline Edad (años) & 0,97 & $0,94-1,01$ & 0,134 \\
\hline \multicolumn{4}{|l|}{ Diagnóstico clínico } \\
\hline Abuso & 1,00 & & \\
\hline Dependencia & 1,12 & $0,58-2,15$ & 0,737 \\
\hline Abuso con patología psiquiátrica & 2,33 & $0,76-7,09$ & 0,137 \\
\hline Dependencia con patología psiquiátrica & 1,63 & $0,67-3,96$ & 0,281 \\
\hline \multicolumn{4}{|l|}{ Tipo de tratamiento } \\
\hline Ambulatorio básico & 1,00 & & \\
\hline Ambulatorio intensivo & 0,60 & $0,22-1,64$ & 0,320 \\
\hline Residencial & 1,23 & $0,52-3,30$ & 0,579 \\
\hline Tiempo de tratamiento (meses) & 0,86 & $0,75-0,98$ & 0,023 \\
\hline Egreso por abandono & 2,39 & $1,12-5,14$ & 0,025 \\
\hline \multicolumn{4}{|l|}{ Puntaje cuestionario de salud general Goldberg } \\
\hline Continua & 1,20 & $1,01-1,42$ & 0,034 \\
\hline Ausencia psicopatología (puntaje 1-4) & 1,00 & & \\
\hline Patología subumbral (puntaje 5-6) & 1,78 & $0,48-6,68$ & 0,171 \\
\hline Presencia psicopatología (puntaje 7-12) & 2,68 & $0,52-13,85$ & 0,241 \\
\hline Estudiar o trabajar en forma estable & 0,49 & $0,25-1,00$ & 0,052 \\
\hline Edad inicio consumo drogas ilegales & 0,99 & $0,95-1,04$ & 0,888 \\
\hline \multicolumn{4}{|l|}{ Droga motivo ingreso a tratamiento } \\
\hline Alcohol & 1,71 & $0,32-9,30$ & 0,532 \\
\hline Marihuana & 1,00 & & \\
\hline Cocaína & 1,00 & $0,18-5,63$ & 1,000 \\
\hline Pasta base de cocaína & 5,44 & $1,12-26,37$ & 0,036 \\
\hline Consumo de alcohol en la familia & 1,00 & $0,43-2,31$ & 1,000 \\
\hline Consumo de drogas ilegales en la familia & 1,24 & $0,60-2,55$ & 0,564 \\
\hline
\end{tabular}

Tabla 4. Apoyo social y su asociación con la recaída del consumo de drogas a los seis meses

\begin{tabular}{|lcrrrrrr|}
\hline & $\begin{array}{c}\text { Odds ratio } \\
\text { crudo }\end{array}$ & IC 95\% & Valor $\mathrm{p}$ & $\begin{array}{c}\text { Odds ratio } \\
\text { ajustado* }\end{array}$ & IC 95\% & Valor $\mathrm{p}$ \\
\hline $\begin{array}{l}\text { Número de amigos íntimos y familiares cercanos } \\
\text { Índice Global Apoyo Social }\end{array}$ & 0,98 & $0,91-1,05$ & 0,498 & $\begin{array}{r}0,97 \\
0,98\end{array}$ & $\begin{array}{l}0,89-1,05 \\
0,96-0,99\end{array}$ & 0,033 & 0,965 \\
\hline
\end{tabular}

*Ajustado por edad, sexo, situación ocupacional, puntaje percepción de salud mental (Goldberg), consumo problemático de alcohol en la familia, consumo de drogas en la familia, tipo de tratamiento y condición de egreso del tratamiento.

modelos por los cuales el apoyo social podría ejercer un efecto protector sobre la salud, existiendo evidencia consistente para ambos ${ }^{12}$. El primer modelo postula que el soporte social tiene un efecto directo en salud, independientemente del nivel de estrés experimentado ${ }^{31}$. El segundo sus- 
tenta una acción amortiguadora (buffer) de la red social frente a potenciales efectos negativos del estrés sobre la salud. En este modelo, el soporte social tiene un efecto sólo cuando la persona experimenta un estresor ${ }^{12,31}$. Asimismo, el apoyo y la red social parecen jugar también una importancia crucial en los comportamientos de riesgo asociados al consumo ${ }^{32,33}$. Sin embargo, es necesaria mayor evidencia empírica respecto a los mecanismos involucrados en el efecto del apoyo social sobre la abstinencia del consumo.

Para evaluar el apoyo social, este estudio utilizó el cuestionario MOS que evalúa el apoyo social percibido. El componente cualitativo de este instrumento, a través de la escala de 19 ítems, mostró un promedio de 78,9 puntos, indicativo de un nivel intermedio de apoyo social, sólo un poco mejor al encontrado en España en drogodependientes que participaron en un programa libre de drogas en prisión (promedio $=67,2)^{22}$. No hemos encontrado en Chile evidencia de otros estudios que midan el grado de apoyo social en este tipo de población.

El componente cuantitativo de este instrumento no resultó ser protector de la recaída de consumo. Aunque en los análisis crudos y ajustados, esta dimensión mostró una tendencia hacia valores indicativos de protección, tales análisis no alcanzaron significación estadística, por lo que es posible que el tamaño muestral no haya sido suficiente para detectar este efecto. Otra explicación, más de fondo, apunta a la validez de esta variable, estrictamente cuantitativa, la cual da cuenta del número de amigos y familiares cercanos que el entrevistado se atribuye, pero no necesariamente traduce la capacidad de tales contactos de proporcionar un soporte social positivo. Más aún si se considera la alta prevalencia de consumo de alcohol y drogas de familiares reportada por los entrevistados.

El alto porcentaje de abstinencia encontrado al sexto mes (71\%), aunque con un menor tiempo de seguimiento, es superior al encontrado en otros estudios que han mostrado tasas de abstinencia entre $40 \%$ y $60 \%$ a un año de seguimiento ${ }^{34}$. El estudio realizado en España por Sánchez-Carbonell mostró asimismo que, a un año de seguimiento de adictos a la heroína que participaron en programas de tratamiento, sólo $51 \%$ de los contactados se mantenía abstinente ${ }^{35}$.
Se encontró que $34 \%$ de los sujetos habían abandonado voluntariamente el tratamiento antes de su finalización. Este grupo mostró, en el análisis univariado, un riesgo más de dos veces superior de recaída que el grupo que egresó por alta terapéutica. Otros estudios han señalado previamente que la permanencia en los programas es predictora de mejores resultados de los mismos ${ }^{36}$.

La baja frecuencia de comorbilidad psiquiátrica, inferior incluso a la reportada en población general ${ }^{37}$, puede ser atribuida a subregistro diagnóstico, problema que hemos reportado con anterioridad en grupos de similares característi$\mathrm{cas}^{38}$. En este estudio constatamos que incluso el diagnóstico de severidad del consumo -variable indispensable en un centro de tratamiento especializado-, no se consignó en $16 \%$ de los pacientes. No obstante, se destaca que los pacientes con logro de abstinencia presentaron menor prevalencia de comorbilidad psiquiátrica en relación a la población no abstinente, lo que puede ser indicativo de la importancia de esta variable en ese $\operatorname{logro}^{16}$ y por cierto, en su persistencia en el tiempo. Por ello, siendo importante su consideración en este análisis, pero conscientes del posible subregistro, se prefirió la utilización de la autopercepción de salud mental que, aunque también tiene limitaciones, al menos mide con igual escala a todos los participantes en el estudio.

Este punto revela una necesidad concreta de capacitación en estos centros, en el diagnóstico y registro adecuado de todas las condiciones relevantes de los pacientes en tratamiento. Esta es una condición básica para una correcta evaluación y para estudios de seguimiento que permitan identificar formas de optimización de los tratamientos.

Una de las fortalezas de este estudio es su carácter longitudinal, que permite apoyar la hipótesis de la dirección de la relación entre apoyo social y recaída del consumo. Además, considera una amplia gama de variables que están asociadas al consumo, escasamente evaluadas en nuestro medio y que debieran ser sometidas a un mayor escrutinio, tales como, la situación laboral, el nivel de percepción de la salud mental o el tipo de egreso del tratamiento. Es destacable asimismo que, a diferencia de otros estudios de evaluación de programas de drogodependencias, donde la diversidad de programas hace muy difícil su 
comparación, los programas de tratamiento financiados por CONACE son relativamente homogéneos en relación a su duración, prestaciones recibidas y perfil de prestadores y usuarios.

Como limitaciones, se destaca que, dada la complejidad causal de las drogodependencias, es posible que otras variables relevantes no hayan sido consideradas o sólo fueron evaluadas parcialmente, lo que conllevaría a un control incompleto de la confusión. Tal puede ser el caso de la patología psiquiátrica, la cual, debido al evidente subregistro, sólo pudo ser considerada indirectamente por su autopercepción. La enfermedad psiquiátrica es un potencial confusor ya que seguramente se asocia tanto con el apoyo social como con la permanencia de la abstinencia. Por lo tanto, su control a través de una variable subrogante, con $76 \%$ de sensibilidad, posiblemente resulta en confusión residual. Otra limitación resulta del reducido tamaño muestral que, aunque suficiente para detectar el efecto, no permite obtener estimadores más precisos ni explorar esta asociación en subgrupos específicos, por edad, género o el tipo de droga que motivó el ingreso a tratamiento.

Finalmente, la variable abstinencia del consumo fue definida a partir del autorreporte de los participantes, lo que podría conllevar cierto grado de error de medición de esta variable. Aunque hay estudios de validación con biomarcadores que han registrado subreporte en población penal ${ }^{39} \mathrm{y}$ en ambientes laborales ${ }^{40}$, no hay estudios previos del subreporte en la población de usuarios de centros de tratamiento sostenidos por CONACE. Es posible que este grupo sujeto a tratamiento, tenga menos problemas en reconocer el consumo de drogas; sin embargo, no se puede descartar que a seis meses de su egreso hayan resurgido las naturales reservas a admitir una conducta no aceptada socialmente. En todo caso, suponemos que el eventual sesgo resultante de la mala clasificación de la abstinencia debiera ser de tipo no diferencial; es decir, que el subreporte ocurre de igual forma en personas con distinto grado de apoyo social. Este tipo de sesgos resulta en una subestimación de la asociación estudiada, por lo que podría especularse que la asociación entre el apoyo social y la reincidencia en el consumo a los 6 meses podría ser aún mayor ${ }^{41}$.

El conocimiento de las variables que ayudan a la prevención de recaídas es un requisito fundamental para el desarrollo de intervenciones en la problemática de las drogodependencias. Hay estudios que muestran que los programas de prevención de recaídas serían más exitosos si incorporaran sistemas de soporte social al interior de sus programas ${ }^{42,43}$. El estudio DATOS-A (Drug Abuse Treatment Outcome Studies for Adolescents), en población adolescente de Estados Unidos de Norteamérica, señaló la importancia de incorporar intervenciones que consideren el funcionamiento interpersonal para mejorar los resultados de los programas de tratamiento 27,29 .

En nuestro conocimiento, éste es el primer estudio que pone de manifiesto la importancia del apoyo social en la recaída del consumo en nuestro país. Deja además buenas interrogantes para futuras investigaciones. Una de ellas es si la definición de la variable respuesta como condición dicotómica de consumo o abstinencia, es el mejor indicador para evaluar un logro terapéutico. En futuras investigaciones sería interesante considerar variables más complejas como la disminución del consumo - un logro en sí mismo- o del nivel de problema derivada del mismo. Además, sería interesante contar con indicadores más objetivos respecto de consumo, esto es, screening de consumo de drogas en muestras biológicas. Asimismo, se debe evaluar la importancia del apoyo social en la recaída del consumo en un seguimiento a más largo plazo de pacientes egresados. Las tasas de recaídas en drogodependientes son elevadas, por lo que es necesaria una perspectiva a largo plazo de la evaluación de los programas de tratamiento ${ }^{44}$. Finalmente, más en relación con el diseño de políticas y programas de salud pública, sería el estudio del impacto que tendría, sobre la disminución de recaídas, la inclusión de estrategias de intervención a nivel de la familia y de la red social de las personas que asisten a programas de tratamiento por consumo problemático de drogas. 


\section{REFERENCIAS}

1. NIDA. Diferentes enfoques de tratamiento para la drogadicción. NIDA Info Facts 2006. Available from: http://www.drugabuse.gov/PDF/InfoFacts/ Metodos06.pdf (Consultado el 15/01/ 2007).

2. NIDA. Principios de Tratamientos para la Drogadicción. 2001 [cited 2007 15/01]; Available from: http://www.drugabuse.gov/PODAT/Spanish/ PODATIndex.html. (Consultado el 15/01/ 2007).

3. Fernández J, SeCADES R. La evaluación de programas de tratamiento para drogodependientes en España. Psicothema 1999; 11: 279-91.

4. Mimer W, Bogenschutz M, Vimarreal M. Investigación y práctica en colaboración: la red de ensayos clínicos del NIDA. Adicciones 2006; 18: 11-22.

5. Bowung A. Social support and social networks: their relationship to the successful and unsuccessful survival of elderly people in the community. An analysis of concepts and a review of the evidence. Fam Pract 1991; 8: 68-83.

6. Совв S. Presidential Address-1976. Social support as a moderator of life stress. Psychosomatic Medicine 1976; 38: 300-14.

7. Совв S. Social support as a moderator of life stress. Psychosom Med 1976; 38: 300-14.

8. De la Revila L, Luna J, Bailón E, Medina I. Validación del cuestionario MOS de apoyo social en atención primaria. Medicina de Familia (And). 2005; 6: 10-8.

9. Haber MG, Cohen JL, Lucas T, Baltes BB. The relationship between self-reported received and perceived social support: A meta-analytic review. Am J Community Psychol 2007; 39: 133-44.

10. REMOR E. Apoyo social y calidad de vida en la infección por el VIH. Aten Primaria 2002. 2002; 30: 143:9.

11. Belón J, Delgado A, Luna J, Lardeu P. Validez y fiabilidad del cuestionario de apoyo social DukeUNC-11. Aten Primaria 1996; 18: 153-63.

12. Cohen S, Wiws TA. Stress, social support, and the buffering hypothesis. Psychol Bull 1985; 98: 31057.

13. Pérez J, Martín F. Normas Técnicas de Prevención NTP 439: El apoyo social. 1997; Available from: http:// www.mtas.es/insht/ntp/ntp_439.htm. (Consultado el 15/09/2006).

14. Sherbourne CD, Stewart AL The MOS social support survey. Soc Sci Med 1991; 32: 705-14.
15. Anderson D, Bilodeau B, Deshaies G, Gilbert M, JoBIN J. [French-Canadian validation of the MOS Social Support Survey]. Can J Cardiol 2005; 21: 867-73.

16. CONACE. Seguimiento de una cohorte de beneficiarios para la evaluación de impacto de los planes de atención de las instituciones en convenio con CONACE-FONASA 2006; Available from: http://www.bibliodrogas.cl/biblioteca/ digital/ Seguimiento_Tratamiento.pdf. (consultado el 27/ 12/2006).

17. GOLDBERG D. The detection of psychiatric illness by questionnaire: London: Oxford University Press; 1972.

18. Lahuerta C, Borrell C, Rodríguez-Sanz M, Pérez K, Nевот M. La influencia de la red social en la salud mental de la población anciana. Gac Sanit 2004; 18: 83-91.

19. Fulierton C, Acuña J, Florenzano R, Cruz C, Weil $\mathrm{K}$. Psicopatología en pacientes hospitalizados en un hospital general. Rev Chil Neuro-Psiquiat 2003; 41: 103-9.

20. Araya R, Wynn R, Lewis G. Comparison of two self administered psychiatric questionnaires (GHQ-12 and SRQ-20) in primary care in Chile. Soc Psychiatry Psychiatr Epidemiol 1992; 27: 168-73.

21. Riquelme N, Merino J. Sistemas de enfrentamiento en familias de enfermos alcohólicos. Cienc enferm 2002 Jun; 8: 37-47.

22. Rodríguez M, Pinzón S, Máiquez A, Herrera J, De Benito M, Cuesta E. ¿Tienen apoyo social y familiar los drogodependientes que participan en el programa dibre de drogas»en prisión? Medicina de Familia (And). 2004; 5: 16-26.

23. De La Reviшa L, Guiшot P. Entrevista semiestructurada sobre apoyo social; Available from: http:// w w w. fundesfam.org/ H tml/ Aprender/ aprender.html (Consultado el 15/10/2006).

24. De la Revilia L, Bailón E, De Dios luna J, Deigado A, Prados M, Frettas L Validación de una escala de apoyo social funcional para su uso en la consulta del médico de familia. Aten Primaria 1991; 8: 688-92.

25. Greentand S, Pearl J, Robins JM. Causal diagrams for epidemiologic research. Epidemiology 1999; 10: $37-48$.

26. De Irala J, Martínez-GonzÁlez M, GuiwÉn F. ¿Qué es una variable de confusión? Med Clin (Barc) 2001; 117: 377-85.

27. Luengo M, VilaA P. Evaluación de la eficacia de los programas de tratamiento de drogodependen- 
cias: un análisis desde los predictores de recaídas. Available from: http://www.drogascadiz.es/ AdminMancLaJanda/UserImages/3df3ba78-fda94590-ae20-5f9e18bfdbd7.pdf (Consultado el 12/ 10/2006)

28. Luengo M, Romero E, Gómez-Fraguela J. La prevención de recaídas en el consumo de drogas: Análisis de la eficacia del programa terapéutico-educativo Proyecto Hombre. Santiago de Compostela: Editorial Compostela; 2001.

29. Galaif E, Hser Y-I, Greua C, Joshi V. Prospective risk factors and treatment outcomes among adolescents in DATOS-A. JAR 2001; 16: 661-78.

30. RHOADs DL. A longitudinal study of life stress and social support among drug abusers. Int J Addict 1983 Feb; 18: 195-222.

31. Ringdal G, Ringdal K, Jordhoy M, KaAsa S. Does social support from family and friends work as a buffer against reactions to stressful life events such as terminal cancer? Palliat Support Care 2007; 5: 61-9.

32. Stein MD, Charuvastra A, Anderson BJ. Social support and zero sharing risk among hazardously drinking injection drug users. J Subst Abuse Treat 2002; 23: 225-30.

33. ZAPKA JG, Stoddard AM, Mccusker J. Social network, support and influence: relationships with drug use and protective AIDS behavior. AIDS Educ Prev 1993; 5: 352-66.

34. Naciones Unidas, Oficina Contra La Droga y el Deцrтo. Por qué invertir en el tratamiento del abuso de drogas: Documento de debate para la formulación de políticas. Available from: http:// www.unod c.org/pdf/report_2003-0131_1_es.pdf. (Consultado el 15/01/2007).

35. Sánchez-Carbonel J, Cam J, Brigos B. Follow-up of heroin addicts in Spain (EMETYST project): resul- ts 1 year after treatment admission. Br J Addict 1988; 83: 1439-48.

36. Toumbourou J, Hamilton M, FaLon B. Treatment level progress and time spent in treatment in the prediction of outcomes following drug-free therapeutic community treatment. Addiction 1998; 93: 1051-64.

37. Vicente B, Rioseco P, Saldivia S, Kohn R, Torres S. [Chilean study on the prevalence of psychiatric disorders (DSM-III-R/CIDI) (ECPP)]. Rev Méd Chile 2002; 130: 527-36.

38. CONACE. Evaluación de proceso y resultado de Planes de tratamiento y Rehabilitación de personas con problemas derivados del consumo de drogas ilícitas en instituciones en convenio con CONACE-FONASA-MINSAL. 2004. Available from: http://www.conace.cl. (Consultado el 15/06/ 2007).

39. FENDRICH M, Xu Y. The validity of drug use reports from juvenile arrestees. Int J Addict 1994; 29: 97185.

40. Cook RF, Bernstein AD, Andrews CM. Assessing drug use in the workplace: a comparison of selfreport, urinalysis, and hair analysis. NIDA research monograph 1997; 167: 247-72.

41. Armstrong B, White E, Saracci R. Principles of Exposure Measurement in Epidemiology. Oxford University Press, New York; 1995.

42. De La Rosa MR, White MS. A review of the role of social support systems in the drug use behavior of Hispanics. J Psychoactive Drugs 2001; 33: 233-40.

43. IRvin JE, Bowers CA, Dunn ME, Wang MC. Efficacy of relapse prevention: a meta-analytic review. J Consult Clin Psychol 1999; 67: 563-70.

44. Grelia CE, Hser YI, Hsieh SC. Predictors of drug treatment re-entry following relapse to cocaine use in DATOS. J Subst Abuse Treat 2003; 25: 145-54. 